\title{
Forward stair descent with hybrid neuroprosthesis after paralysis: Single case study demonstrating feasibility
}

\author{
Thomas C. Bulea, PhD; ${ }^{1-2 *}$ Rudi Kobetic, MS; ${ }^{2}$ Musa L. Audu, PhD; ${ }^{1}$ John R. Schnellenberger, MS; ${ }^{2}$ Gilles \\ Pinault, MD; ${ }^{2}$ Ronald J. Triolo, $\mathbf{P h D}^{1-3}$ \\ ${ }^{1}$ Department of Biomedical Engineering, Case Western Reserve University, Cleveland, $\mathrm{OH} ;{ }^{2}$ Louis Stokes Cleveland \\ Department of Veterans Affairs Medical Center, Cleveland, $\mathrm{OH} ;{ }^{3}$ Department of Orthopaedics, Case Western Reserve \\ University, Cleveland, $\mathrm{OH}$
}

\begin{abstract}
The ability to negotiate stairs is important for community access and independent mobility but requires more effort and strength than level walking. For this reason, previous attempts to utilize functional neuromuscular stimulation (FNS) to restore stair navigation after spinal cord injury (SCI) have had limited success and are not readily generalizable. Stair descent is particularly challenging because it requires energy absorption via eccentric muscle contractions, a task not easily accomplished with FNS. This article presents the design and initial testing of a hybrid neuroprosthesis with a variable impedance knee mechanism (VIKM-HNP) for stair descent. Using a 16-channel percutaneous FNS system, a muscle activation pattern was synthesized to descend stairs with the VIKMHNP in a step-by-step fashion. A finite state control system was implemented to deactivate knee extensor stimulation and utilize the VIKM-HNP to absorb energy and regulate descent speed. Feasibility testing was performed on one individual with complete thoracic-level SCI. Stair descent was achieved with maximum upper-limb forces of less than $45 \%$ body weight compared with previously reported value of $70 \%$ with FNS only. The experiments also provided insight into design requirements for future hybrid systems for stair navigation, the implications of which are discussed.
\end{abstract}

Key words: assistive technology, bracing, finite state control, functional electrical stimulation, functional neuromuscular stimulation, hybrid orthosis, neuroprosthetics, rehabilitation engineering, spinal cord injury, stair descent.

\section{INTRODUCTION}

The ability to negotiate stairs is important to unencumbered community ambulation. For those with lowerlimb paralysis, restoration of stair navigation can expand independence by providing access to non-wheelchair friendly environments. The success of neuroprosthetic systems in restoring standing and walking function on level surfaces [1-4] further support extension of similar interventions for stairs. But stair navigation is quite different and generally speaking more demanding than level walking. Stair ascent and descent differ from normal gait in many ways, including joint ranges of motion, relative timing of muscle activation, contact forces, joint

\footnotetext{
Abbreviations: AFO $=$ ankle-foot orthosis, $\mathrm{CL}=$ controlled lowering phase, EMG = electromyography, FNS = functional neuromuscular stimulation, FSR = force sensitive resistor, GUI = graphical user interface, HNP = hybrid neuroprosthesis, IPI = interpulse interval, LO = leg off phase, LP = leg pull through phase, $\mathrm{PW}=$ pulse width, $\mathrm{PWM}=$ pulse width modulation, $\mathrm{SCI}=$ spinal cord injury, VIKM = variable impedance knee mechanism, VIKM-HNP = hybrid neuroprosthesis including a variable impedance knee mechanism.

*Address all correspondence to Thomas C. Bulea, PhD; Building 10, CRC, Room 1-1469, 10 Center Dr, MSC-1604, Bethesda, MD 20892; 301-451-7533.

Email: thomas.bulea@nih.gov

http://dx.doi.org/10.1682/JRRD.2013.12.0257
} 
moments, and power generation and absorption. Stair ascent requires concentric contractions of lower-limb muscles in combination with upper-limb assistance to propel the body upward and forward in a stable manner. In contrast, stair descent requires a person to control the intrinsic force of gravity. As a result, stair ascent requires generation of considerable internal energy (via concentric muscle contraction), whereas descent necessitates power absorption, which is achieved through eccentric muscle contractions.

Functional neuromuscular stimulation (FNS) is well suited for generation of concentric muscle contractions and has been employed in stair climbing systems previously with some success [5-8]. Although FNS-assisted stair ascent is possible, all reported systems exhibit insufficient stimulated lower-limb forces to independently overcome gravity, bring the body forward, and provide stability. These deficiencies in concentric contractile strength can be effectively compensated for with modest upper-limb effort. Although possible in insolated muscle preparations [9], eccentric contractions are difficult to elicit and control through stimulation in clinical situations [10]. Thus, users of FNS systems find stair descent to be a frightening experience that results in almost exclusive reliance on upper-limb effort to lower the body and complete the maneuver [6].

Traditionally, stair descent has been examined as a step-over-step cycle with no double support phase on the same level [11-14]. In a similar manner as walking, stepover-step stair descent in nondisabled individuals is typically divided into distinct phases based on actions of each limb, namely weight acceptance, forward continuance, controlled lowering, leg pull through, and foot placement [12]. The first three phases constitute stance, with weight acceptance representing a period of double support and power absorption at the knee and ankle. Forward continuance represents the transition to single support as the body moves forward. Controlled lowering encompasses the end of single support; it is the major portion of progression from one step to the next that requires power absorption at the knee, and to a lesser extent, the ankle. Leg pull through follows controlled lowering and consists of power generation for hip and knee flexion and ankle plantar flexion to lift the now trailing limb from the step. Hip flexion continues until midswing, when hip and knee flexion is reversed to extension while the ankle is plantar flexed to facilitate foot placement.
Altering the gait pattern so both feet are placed on each step creates a slower, safer, and more static descent. This controlled stepping strategy limits the necessity of forward continuance of the body center of mass, essentially reducing stair descent into a problem of leg off, controlled lowering, weight acceptance, and leg pull through. In normal descent, weight acceptance is dominated by power absorption at the ankle, resulting in peak joint moments and power absorption similar to those of walking [11-12,14]. Controlled lowering requires considerable energy dissipation at the knee, with knee flexion moments up to three times those experienced during normal walking and knee power for energy absorption up to five times normal walking levels [11-14]. These periods of high torque and power absorption occur when the knee is flexing; thus, a large, eccentric contraction of knee extensors is required to prevent leg buckling.

We have previously introduced an orthotic device that provides variable stiffness at the knee joint to overcome the challenges of controlling eccentric contractions with stimulation [15]. This device, termed a variable impedance knee mechanism (VIKM), has the capability to dissipate energy at the knee joint while simultaneously allowing knee flexion. The VIKM uses a magnetorheological damper to provide controllable resistance to knee motion. It is a passive device that can automatically adjust stiffness to lock the knee joint or to dissipate energy during motion [15]. We have combined the VIKM with an implanted FNS system to create a hybrid neuroprosthesis (HNP) including a VIKM (VIKM-HNP) that restores walking following paralysis from spinal cord injury (SCI) [16]. The FNS system was composed of 16 intramuscular electrodes with percutaneous leads, implanted bilaterally in muscles for controlling hip flexion (sartorius, tensor fasciae latae, gracilis) and extension (gluteus maximus, posterior portion of adductor magnus), knee flexion (sartorius, gracilis) and extension (vastus medialis, vastus intermedius, and vastus lateralis), and ankle dorsiflexion (tibialis anterior). In the case of knee extension, single electrodes recruited multiple muscles of quadriceps excluding rectus femoris [16]; these electrodes are referred to as left and right quadriceps. During walking, coordinated stimulation of muscles propels the body forward while the VIKM helps to control knee motion during stance and swing phase. Our previous studies have demonstrated the benefits of the VIKMHNP for ambulation compared with FNS-only systems, namely, the ability to replace the function of eccentric 
knee extensors to absorb power and restore knee flexion during stance phase [17]. We hypothesized that power dissipation at the knee, accomplished by the VIKM-HNP, could enable consistent FNS-driven stair descent. A properly designed HNP for stair descent should be able to excite the paralyzed muscles to generate power for leg off and leg pull through without resistance and activate the VIKM when appropriate to absorb energy during controlled lowering and weight acceptance. This report summarizes the design of a novel finite state control system to achieve forward stair descent with an HNP containing the VIKM. Feasibility testing of the system during stair descent with one individual with thoraciclevel SCI is also presented.

\section{METHODS}

\section{Development of Functional Neuromuscular Stimulation Descent Pattern}

One individual was recruited to evaluate stair descent feasibility with the VIKM-HNP. He consented to participate as approved by the institutional review board of the Louis Stokes Cleveland Department of Veterans Affairs Medical Center. The study volunteer was a $50 \mathrm{yr}$ old male, $1.74 \mathrm{~m}$ in height, and $62 \mathrm{~kg}$ in weight with an American Spinal Cord Injury Impairment Scale A motor and sensory complete SCI; the level of neurological injury was T7 (thoracic 7). He was 27 yr postinjury and was implanted with a 16-channel percutaneous stimulation system with intramuscular electrodes to facilitate standing and walking. The participant had used his implanted FNS system regularly for standing, walking, and exercise to maintain muscle mass and strength over a period of $20 \mathrm{yr}$. A baseline pattern for walking with the VIKM-HNP was described previously [16].

Prior studies of electromyography (EMG)-based muscle activity during stair descent indicate that there are significant differences from walking. Specifically, increased quadriceps activity was observed during single-support phases for power absorption, signifying the presence of eccentric contractions [12], while the relative duration of knee flexor activation was reduced compared with level walking [11]. Muscle activity and the resultant moments at the hip have been reported to be variable across subjects because of changes in trunk position and speed of descent [11-13,18-19]. In general, hip extensor activity is required during the majority of stance phase of step-over-step descent to counteract external flexion moment, with the exception of the period just before toe-off, when hip flexors are usually activated [19].

To synthesize stair descent using FNS, the maneuver was redefined to accommodate for the reduced muscle set accessible to stimulation and the capabilities of the VIKM orthosis. A step-by-step strategy was employed whereby both feet are placed on each step, a change that can decrease the joint forces and moments by up to 50 percent compared with step-over-step descent [11]. The reduced muscle set and step-by-step strategy required changes to the typical phases of descent observed in nondisabled individuals. First, forward continuance phase was replaced by a leg off phase (LO), which consisted of hip and knee flexion to lift the leading limb from the step. Second, the leg pull through phase (LP) was expanded to include the foot placement phase because the trailing limb did not advance beyond the leading limb. Thus, the FNS-driven descent cycle was shortened to four phases: LO, controlled lowering phase (CL), weight acceptance, and LP (Figure 1).

The subject-specific stimulation pattern for stair descent is shown in Figure 2. Note that the hip and knee joints were under control of FNS, while the ankles remained locked by ankle-foot orthoses (AFOs) throughout descent. In this implementation of the VIKM-HNP, knee extensor stimulation was not active during CL, the major period of knee power absorption. Instead, controlled lowering was achieved through activation of the VIKM. Furthermore, lower-limb motion was restricted to the sagittal plane by the orthosis. The level of muscle activation was controlled by two parameters: stimulus pulse width (PW) and interpulse interval (IPI), the inverse of frequency. The biphasic stimulation was current controlled with an amplitude of $20 \mathrm{~mA}$, and maximum stimulus PW was limited to $150 \mu$ s to prevent tissue damage [20]. The maximum stimulation frequency was set at $33.33 \mathrm{~Hz}$ (IPI = $0.03 \mathrm{~s}$ ) to minimize fatigue [21]. When less muscle strength was required, the IPI was increased to $0.06 \mathrm{~s}$ to deliver stimulation at $16.67 \mathrm{~Hz}$. The periods of high and low frequency are denoted in Figure 2 by black and light gray areas, respectively.

The stimulation pattern in Figure 2 was generated using a procedure similar to one previously developed for walking [2]. Briefly, a template pattern of muscle activation was developed based on normal EMG activity during stair descent and previously established rules for walking with electrical stimulation [2]. The template was 
JRRD, Volume 51, Number 7, 2014

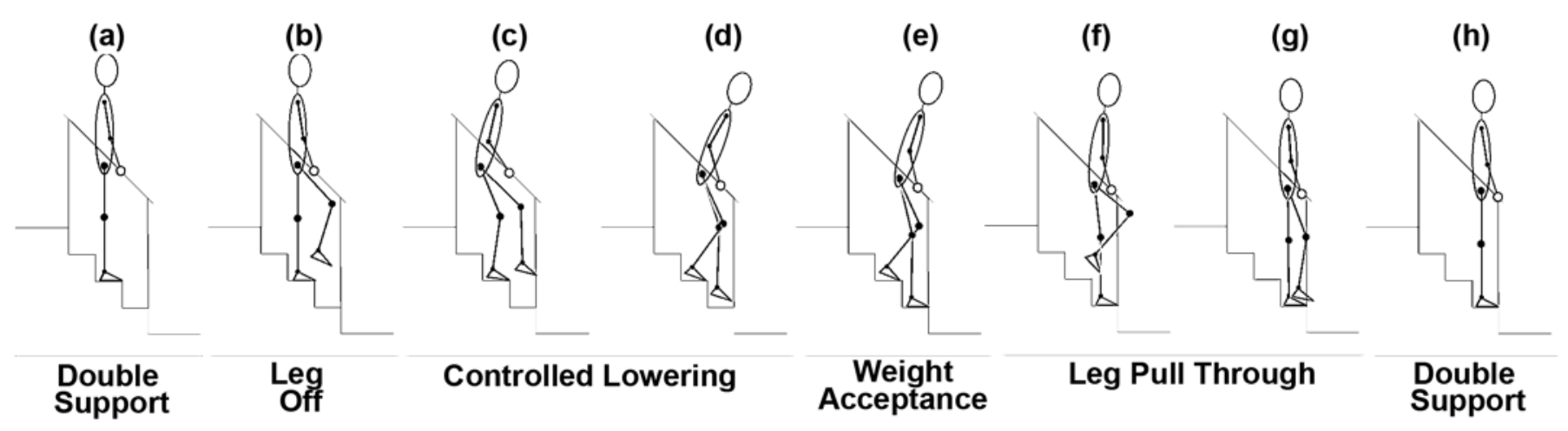

Figure 1.

Step-by-step stair descent achieved by functional neuromuscular stimulation, including description of phases: (a) double support, (b) leg off, (c)-(d) controlled lowering, (e) weight acceptance, (f)-(g) leg pull through, and (h) double support. Right limb begins descent cycle (leading) while left limb ends it (trailing).

tailored to the participant by adjusting the stimulation parameters (PW and IPI) based on the recruitment properties of each muscle. A manual muscle test was used to establish the maximum PW for each muscle by observing the point at which spillover to adjacent muscles occurred (subject to the safety limit of $150 \mu \mathrm{s}$ ). Stair descent was divided into five phases comprising distinct movements at each joint within each phase (Figure 1). The stimulation level of each muscle was increased or decreased to achieve the desired motion within each phase based on visual observation of stair descent trials in which the subject was supported by a spotter to ensure safety. If the intensity of stimulation was to be increased, the strategy was to first maximize PW and then decrease IPI (thereby increasing frequency). The relative timing of muscle activation and deactivation was also adjusted based on visual inspection. The resulting pattern of descent is shown in Figure 2, while muscle activity during each phase is described in detail subsequently.

Stair descent was initiated from quiet standing during double support (Figure 1(a)). LO was triggered by the user with a finger switch and began with hip and knee flexion under FNS control (Figure 1(b)). This relatively short period of flexion was followed by automatic activation of the lead leg knee extensors coincident with deactivation of ipsilateral hip and knee flexors and contralateral hip and knee extensors (Figure 1(c)). This pattern extended the lead limb to ensure the foot cleared the current step while simultaneously allowing flexion of the trailing limb to lower the body to the next step. Lead limb hip extensors were automatically activated as hip flexors turned off (Figure 1(d)), preparing the limb for loading after ground contact. This delay in hip extensor activation, combined with delayed hip flexor deactivation, ensured that the lead limb did not contact the front of the prior step as it lowered to the next one. Trailing limb hip extensors were activated shortly after leading limb hip extensors to prevent forward trunk tilt and aid in stability after weight acceptance (Figure 1(e)). When the double support phase was achieved after weight acceptance, the pattern stopped until a second input was received from the manual finger switch. Once this occurred, trailing limb hip extensors were ramped off as knee and hip flexors were turned on to facilitate the first phase of LP (Figure 1(f)). Similar to LO, this flexion period lasted for a short time, after which hip and knee extensors were activated while flexors were deactivated, allowing extension of the trailing limb prior to double support (Figure 1(g)). At this point, knee and hip extensors remained on bilaterally to provide body weight support during double stance (Figure 1(h)), returning to the initial state in the sequence in preparation for descent to the next step.

\section{Stair Descent Finite State Machine}

The stair descent cycle achieved by the synthesized FNS pattern was split into three distinct phases for statebased control purposes: LO, CL, and LP. Similar to walking [16,22], the distinct behavior of the knee during these phases suggested that a finite state machine can be implemented to control the VIKM during stair descent. The 


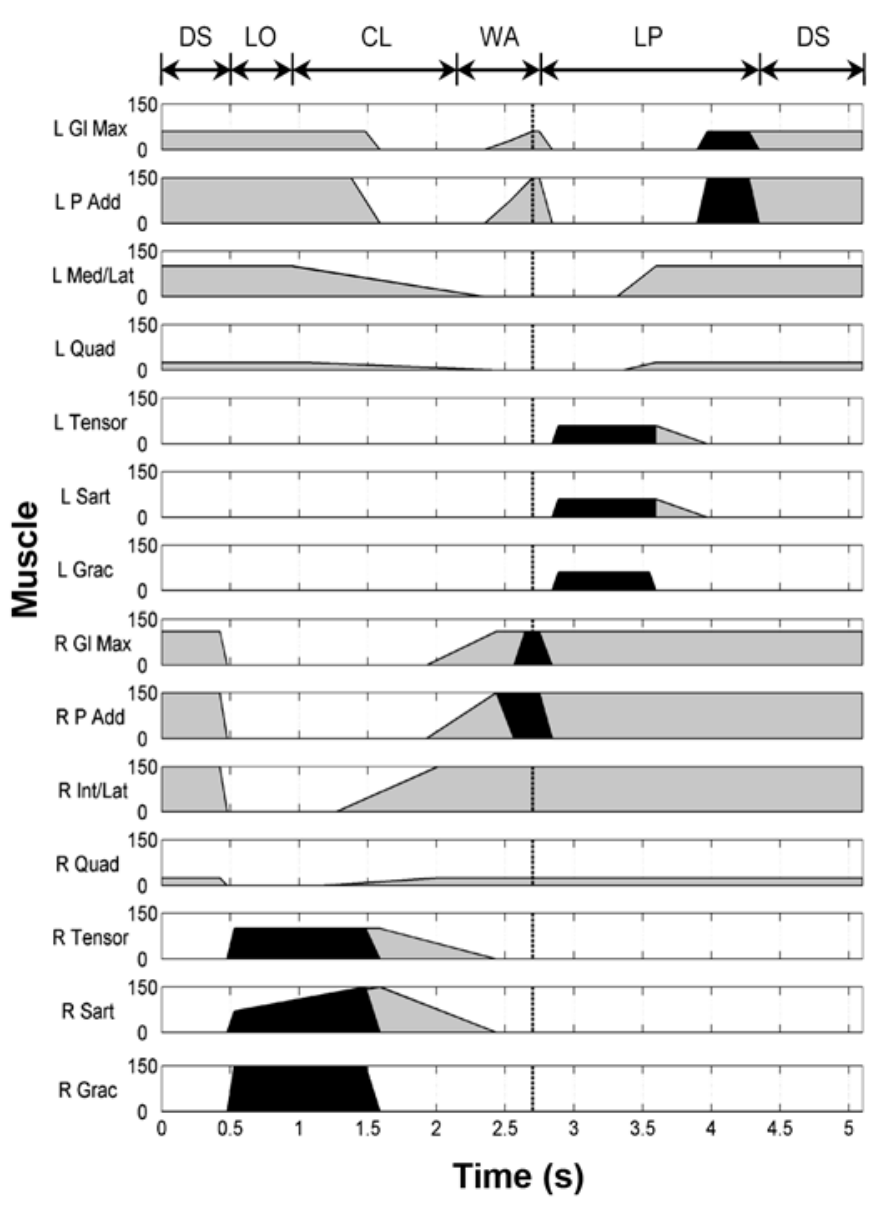

Figure 2.

Synthesized 14-channel stimulation pattern for stair descent with hybrid neuroprostheses including variable impedance knee mechanism as function of time. Phases of stair descent (Figure 1) are indicated on top of figure. Stimulus pulse width (in microseconds) of each muscle is plotted. Shading represents two frequencies of stimulus pulse train, $33.33 \mathrm{~Hz}$ (black) and $16.67 \mathrm{~Hz}$ (gray). Pattern is triggered by user pressing finger switch; dotted line $(-2.7 \mathrm{~s})$ is point at which stimulation is held between states 2 and 3 while waiting for second finger switch input (see Figure 3 ). Electrodes for stimulation of tibialis anterior (TA) were implanted bilaterally [16]; however, ankles were locked using ankle-foot orthoses for stair descent and thus TA stimulation was omitted. $\mathrm{CL}=$ controlled lowering, $\mathrm{DS}=$ double support, $\mathrm{GI}$ Max = gluteus maximus, Grac = gracilis, Int = vastus intermedius, $L=$ left, Lat = vastus lateralis, LO = leg off, LP = leg pull through, Med = vastus medialis, $\mathrm{P}$ Add = posterior portion of adductor magnus, $\mathrm{Quad}=$ quadriceps, $\mathrm{R}=$ right, Sart = sartorius, Tensor = tensor fasciae latae, WA = weight acceptance. purpose of the VIKM-HNP stair descent finite state machine was to coordinate the actions of the VIKM orthosis with those elicited by stimulation. The objective was to activate the VIKM only when power must be absorbed by the knee joint (i.e., CL). The VIKM was to remain inactive when limb motion was to be driven by FNS (i.e., LO and LP) so as not to impede those phases of the maneuver.

The stair descent finite state machine transitioned between states using feedback from brace-mounted sensors, a user finger switch, and the FNS pattern. Conceptual operation of the controller for stair descent led by the right leg proceeded as follows (Figure 3). The user operated a finger switch with two active buttons to scroll through a menu of options. This menu included stimulation patterns for sit-to-stand transition, quiet standing, walking, stair descent, and stand-to-sit transition. When at the top of a staircase, the user selected the stair descent mode of operation, which automatically placed the controller in quiet stance (state 0). Descent was initiated when the user pressed the manual switch, which transitioned the controller to state 1 (LO). The VIKM damper and knee extensor stimulation of the leading (right) limb were turned off to allow knee and hip flexion under FNS control, while the trailing limb (left) VIKM damper and knee extensors stimulation were turned on to provide support during single stance. When the right knee extensor stimulation was activated - the right foot was off the ground - the controller transitioned to state 2 (CL). During this phase, right knee extensors remained on while the damper was turned off to aid in unencumbered foot placement on the step below. The left knee extensor muscles were turned off while the damper was activated to allow controlled knee flexion to lower the body to the next step. Once the right foot hit the ground, the right VIKM damper was activated for weight support and the finite state machine waited for a second input from the user finger switch. During this time, left knee extensor stimulation remained off and left VIKM damper activity was held constant to create a static posture with the feet on both steps. When triggered, the controller entered state 3 (LP). The right VIKM was active to aid in single support while the left VIKM was deactivated as the trailing limb flexed under FNS control. When left foot contact was sensed, the controller re-entered state 0 (double support). From this state, the finger switch was used to either trigger another step of stair descent or change modes to level walking if the bottom of the stairs had been reached. 


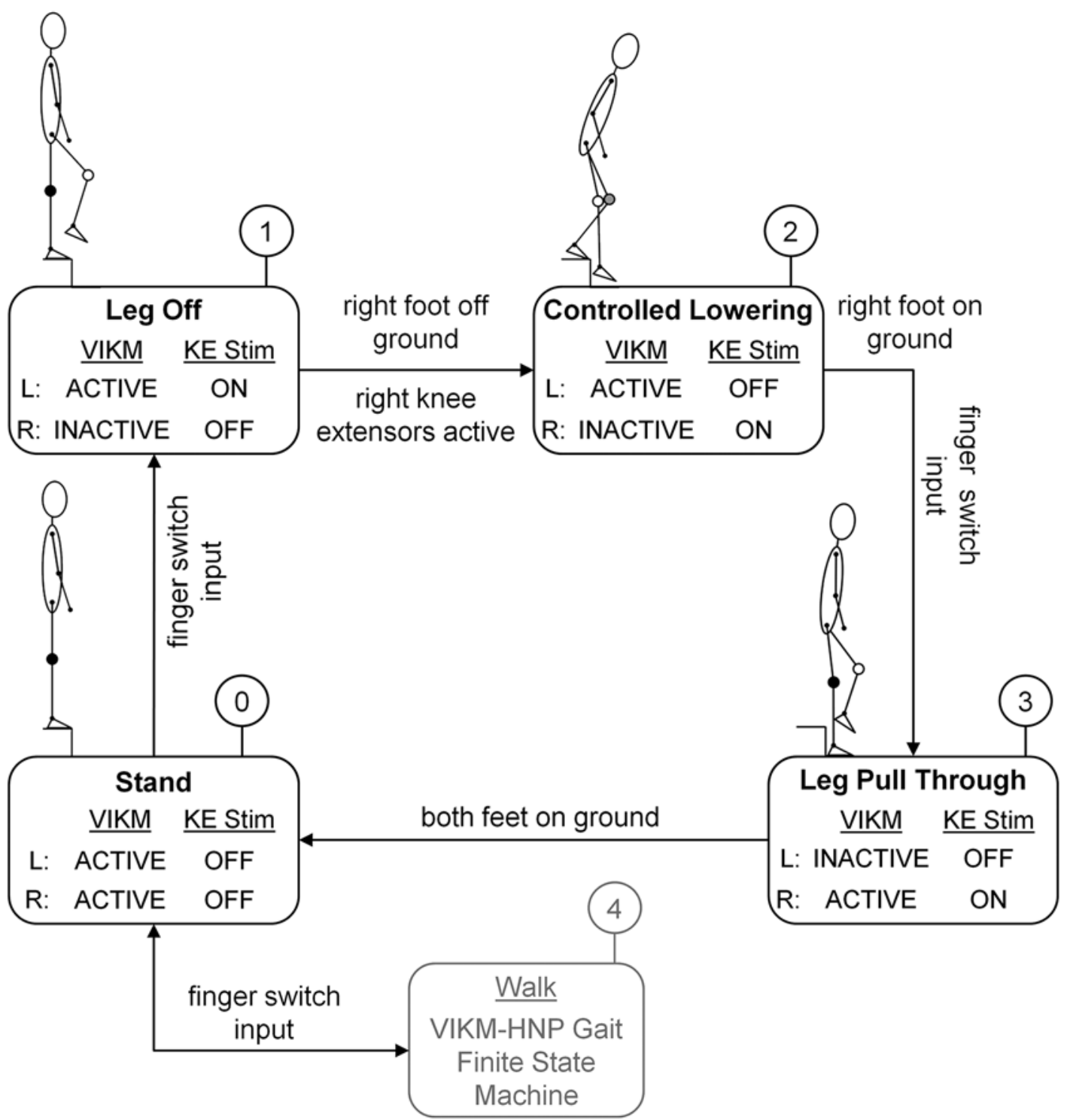

Figure 3.

Hybrid neuroprosthesis including variable impedance knee mechanism (VIKM-HNP) finite state machine showing status of leading right $(\mathrm{R})$ and trailing left (L) limb VIKM damper and knee extensor stimulation (KE Stim) within each state with transition between states indicated. Note that only knee extensor muscles are modulated by controller; all other muscles remain unaffected by finite state machine. Above each state, resistance level of VIKM for leading (R) and trailing (L) knee is depicted in schematic. Black circle indicates highest duty cycle (lock knee to prevent motion), gray circle indicates moderate duty cycle to regulate knee motion, and white circle indicates no activity to allow unencumbered knee motion.

The VIKM is capable of providing eight distinct levels of resistance to knee motion [15-16]. Note that during periods of static single support-both legs in state 0 , the trailing limb in state 1 and the leading limb in state 3-the VIKM was active at the highest level to prevent knee motion. During the power absorption period of state 2, the VIKM on the trailing limb was active at a less than maxi- mal level to facilitate knee flexion for controlled lowering. Unlike the finite state machine for level walking [16], the stair descent controller did not automatically adjust VIKM resistance to knee motion. Instead, the level of resistance during CL was set before each descent trial and held constant during each step down. This mode of operation assured safety during controlled lowering. Prior to the 
experimental trials, calibration trials of stair descent were performed with a spotter present to establish the resistance level of the VIKM based on user feedback regarding comfort with the speed of descent. Previous studies indicated that peak knee extensor moment was approximately $59 \mathrm{Nm}$ when descending to the floor with a handrail [11], a maneuver similar to step-by-step descent studied here. Starting at a resistive torque above this level, the setting was incrementally decreased until the user was no longer comfortable with the speed of lowering (based on verbal feedback). Two levels of VIKM resistance for CL were identified from these calibration trials, providing average torques of 47 and $38 \mathrm{Nm}$ at $90 \%$ [16]. These levels were chosen because the resistive torques were large enough to ensure user comfort with the lowering speed yet different enough to evaluate the capacity of the VIKM to control the speed of lowering the body to the next step. The subject was blinded to which VIKM resistance setting was employed during testing.

\section{Implementation}

The stair descent control system was implemented on a prototype VIKM-HNP for feasibility testing. A sensor set consisting of potentiometers (ALPS Electric; Campbell, California) and force sensitive resistors (FSRs) (B\&L Engineering; Tustin, California) monitored hip and knee angles and foot-ground contact, respectively. Joint angular velocity was determined by differentiating the angle measurements. Each foot contained four FSRs, one each at the first phalange, the first and fifth metatarsals, and the heel. The signals from the three FSRs in the forefoot were summed with equal weights to create a single signal representing the forefoot. Foot contact was sensed when the output of either the forefoot or heel signal exceeded 90 percent of their value during quiet stance. The sensors were powered by a rechargeable Sony NPF970 lithium ion battery (7.2-8.4 V, 47.5 Wh, 6,600 $\mathrm{mAh}$ ) (Tokyo, Japan). All sensor signals were collected at $200 \mathrm{~Hz}$ and low-pass filtered using fifth-order Butterworth filters with cutoff frequencies of $10 \mathrm{~Hz}$ for the potentiometers and $20 \mathrm{~Hz}$ for the FSRs. A PW modulation (PWM) drive circuitry was developed to power each VIKM using a Sony NP-F570 lithium ion battery (7.28.4 V, 15.8 Wh, 2,200 mAh). The drive circuitry adjusted the resistance of each VIKM by setting the duty cycle of a PWM drive signal as indicated by a digital output from the controller [16].
The VIKM-HNP stair descent control system was realized using the xPC target environment (MathWorks Inc; Natick, Massachusetts). The target computer ran the control software program in real time and collected and logged data through two data acquisition cards (National Instruments; Austin, Texas) containing multiple analog input and digital input/output channels. The host computer ran a graphical user interface (GUI) to control the target application. The GUI was an integral part of the system since it enabled an operator to calibrate sensors, adjust controller thresholds, set VIKM resistance level, and determine which mode of locomotion was being used (e.g., stand, level walking, stair descent). The xPC target was connected to a universal external control unit, which delivered stimulation to the muscles of the lower limb using the synthesized pattern for stair descent.

\section{Experimental Setup}

In agreement with previously published studies [58], we observed that forward stair descent with FNS-only was erratic. It was also uncomfortable for the participant because of the difficulty experienced attempting to control body lowering by deactivating trailing limb hip and knee extensors, leading to heavy reliance on arm support. As a result, the focus of our experimental evaluation was on the feasibility of the VIKM-HNP to control lowering of the body through regulating knee flexion. This was accomplished by starting at the highest damping level that would allow smooth motion and then decreasing incrementally. The limb motion required for stair descent was achieved using the profiled stimulation pattern.

Because a step-by-step strategy was employed, an experimental staircase composed of a single step was constructed by positioning a platform 8 in. above the floor (Figure 4). The platform was 36 in. wide and 72 in. deep, which allowed the user space to stand and position himself for descent. Horizontal lines were placed on the platform and floor below to indicate a tread of 9.5 in., creating an effective staircase slope of $40^{\circ}$, which is similar to previous studies [12-13]. Two force plates (AMTI Inc; Watertown, Massachusetts) were used to collect ground reaction forces. One was placed in a cutout section in the center of the platform at the step edge, and the other was in the floor. Two handrails were mounted on either side of the platform at the same slope as the step to provide additional support. Each handrail was instrumented with two six-axis load cells (AMTI Inc) to measure the upper-limb forces during descent. Markers were placed on the user and the VIKM-HNP orthosis using a 
specific setup specially designed to accommodate the brace [23]. A 16-camera Vicon MX40 motion capture system (Vicon Inc; Oxford, United Kingdom) was used to measure trunk and lower-limb kinematics. All data were sampled at $200 \mathrm{~Hz}$.

During the experimental trials, the participant wore the prototype VIKM-HNP. For safety, a handle was placed on the back of the trunk corset and a spotter remained in close proximity to the subject at all times to provide extra support if needed (Figure 4). The hips remained free to move under FNS control, the knees were controlled using the VIKM orthosis, and the ankles remained locked using AFOs. Each trial consisted of descending a single step followed by a few steps of walking and then a short rest (30-60 s) before another trial. Using a wheelchair and ramp, the participant positioned himself on the top platform and stood using the finger switch to trigger the FNS pattern for sit-to-stand transition. Stair descent was then triggered using the manual

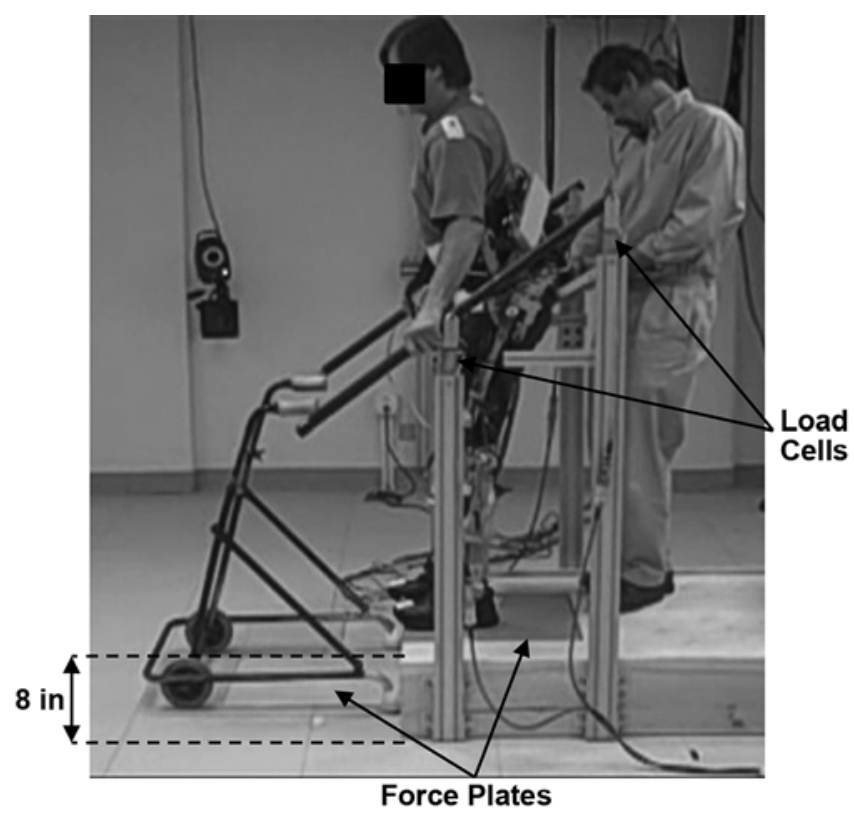

Figure 4.

Experimental setup for evaluation of stair descent with hybrid neuroprosthesis including variable impedance knee mechanism. Four six-axis load cells were placed below mounting points of hand rails to monitor upper-limb force. Force platforms were installed at each level to measure ground reaction forces. Once user completed descent, he transferred support to walker and stepped away from platform to sit. Spotter was present to ensure safety. finger switch, first to initiate $\mathrm{LO}$ and $\mathrm{CL}$ and second to trigger LP. After one step of descent, the participant transferred his arms to a walker and selected a walk pattern to move away from the stair platform prior to sitting. The resistance level of the VIKM during CL was set by an operator using the GUI interface on the host computer before each stair descent trial. Operation of the VIKMHNP during walking was achieved using the previously developed level ground control system [16]. A total of 14 stair descent trials were performed; 6 at the higher torque level of the VIKM (47 Nm) and 8 at the lower level (38 Nm). All data were collected and analyzed offline. For comparison between trials, a one-way analysis of variance with a 95 percent confidence interval was used to determine statistical significance.

\section{RESULTS}

\section{Limb Motion}

While the participant was able to achieve forward stair descent using FNS only, the absence of power dissipation capability resulted in erratic, inconsistent descent with heavy reliance on arm support. The FNS maneuver was uncomfortable and unstable, and thus, we did not perform a full set of testing with only FNS. Unlike with FNS only, the participant was able to achieve consistent forward stair descent using the VIKM-HNP. Lower-limb joint angles and foot contact on-off times for a typical stair descent cycle are shown in Figure 5. In this and all subsequent descent cycles, the right limb was leading and the left limb was trailing. The descent cycle began in double support phase, during which both knees were near full extension while both hips were flexed to approximately $30^{\circ}$. This flexion was a result of forward trunk tilt by the participant, which enabled him to see the step below. Forefoot signals for both feet were zero during this phase since the user's posture-locked ankle and fully extended knees-applied most of the body weight through the heel. The transition to LO was achieved by pressing the manual finger switch, which activated right knee and hip flexors, resulting in the right heel leaving the step. Knee flexion to approximately $30^{\circ}$ —which was aided by gravity - occurred rapidly, while antigravity hip flexion was slower to react to stimulation. Hip flexion during the end of LO was also aided by counterclockwise trunk rotation and trunk extension. Approximately $1 \mathrm{~s}$ after flexion was initiated, right limb knee extensors were activated, which triggered the transition to CL. In this 
phase, left limb hip and knee extensors were deactivated while the VIKM became active to regulate knee flexion. Rapid coincident hip and knee flexion were observed, which caused the left heel to rise from the step as weight was transferred to the front of the foot via the forefoot rocker mechanism. Left leg flexion, combined with right knee extension, brought the right heel to the floor. At this point, stimulation and VIKM states were held constant, creating a stable posture until a second finger switch input was received (Figure 5). During this time, left and right hip extensors were active to prevent forward collapse of the trunk. The subject used his upper limbs to assist weight transfer to the leading limb, resulting in bilateral hip extension. After the finger switch was pressed for a second time ( 3.5 s), left hip and knee flexors were activated to initiate LP. In most trials, stimulated
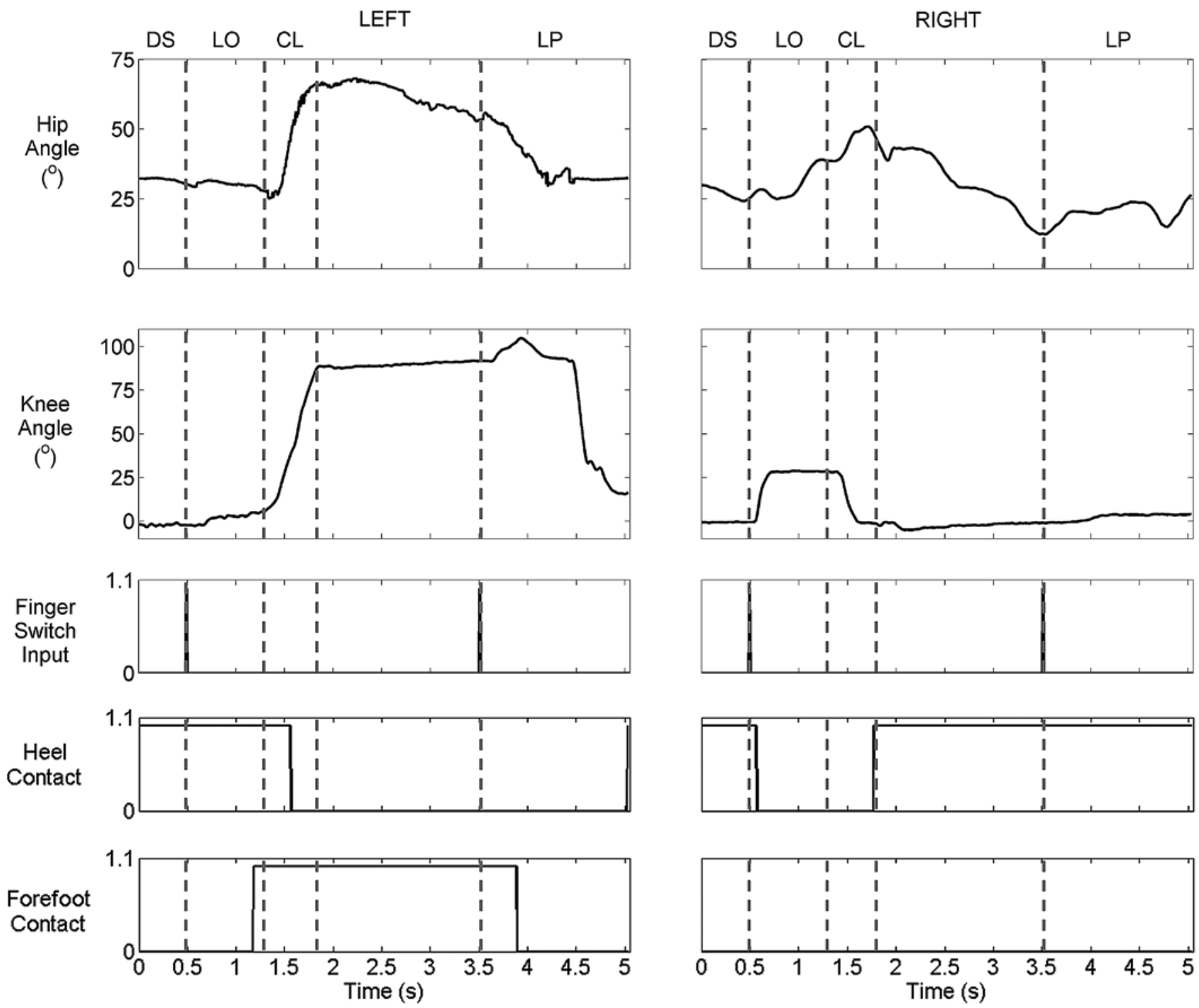

Figure 5.

Left (trailing) and right (leading) limb sagittal joint angles, finger switch input, and left and right foot contact for typical stair descent cycle with hybrid neuroprosthesis including variable impedance knee mechanism. CL = controlled lowering, DS = double support, LO = leg off, LP = leg pull through. 
flexor contractions were not strong enough to fully clear the step, and a compensatory mechanism was observed whereby the user initiated hip extension via trunk extension and clockwise trunk rotation in the transverse plane, which enabled the user to swing the trailing limb toward the ground. This mechanism was aided by the automatic activation of left limb knee and hip extensors for placement of the trailing foot on the ground heel first, completing the descent cycle.

\section{Controlled Lowering Regulated by Variable Impedance Knee Mechanism}

The ability of the VIKM to regulate knee motion during the lowering task was evaluated by comparing stair descent trials performed with two distinct levels of VIKM resistance applied during CL. One set of trials was performed using a VIKM duty cycle of 51.2 percent, while a second set of trials was performed with the VIKM at 39.2 percent duty cycle (Table). These levels of VIKM activation-which were determined during calibration trials as described previously-provided an average resistance of 47 and $38 \mathrm{Nm}$, respectively. The resistance level was randomized, and the participant was blinded to the level of resistance in a given trial. Figure 6 shows typical results for stair descent with the high (solid line) and low (dashed line) VIKM duty cycle applied during controlled lowering. The transition from LO to CL occurred at approximately $1.2 \mathrm{~s}$ when the right knee extensor stimulation became active and the VIKM transitioned to the preset duty cycle for lowering. Deactivation of left knee extensors occurred after the VIKM duty cycle was set, at approximately $1.4 \mathrm{~s}$. A distinct difference in behavior between the two trials was observed during this phase; these differences are summarized in the Table.

The duration of CL, which started with left VIKM duty cycle transition and ended when left knee angle reaches steady-state, was significantly longer during trials at the higher duty cycle. The average and peak left knee angular velocities were also significantly increased at the lower VIKM duty cycle (Table). The user was able to perceive the difference in CL speed, consistently stating preference for the lower speed, higher duty cycle setting.

The forces measured from the hand rails and the force platforms during the controlled lowering phase of stair descent for a typical trial are shown in Figures $\mathbf{7}$ and $\mathbf{8}$. The forces were normalized by the combined weight of the participant and the VIKM orthosis (74.1 kg). At the beginning of knee flexion (approximately $1.4 \mathrm{~s}$ ) the vertical support provided by the hands was almost zero, indicating the participant's trust in the VIKM orthosis to regulate knee motion and allow his body to be lowered to the next step. As a result, a rapid increase in knee flexion velocity was observed (Figure 6). This velocity increase caused the participant to resume body weight support using the upper limbs, resulting in a slight decrease in knee flexion velocity at approximately $1.6 \mathrm{~s}$. The vertical support forces at this point were larger during descent with VIKM duty cycle of 39.2 percent compared with 51.2 percent, but the knee flexion velocity was faster at the lower duty cycle. A shift in the horizontal force profile was observed after the spike in vertical arm support during the faster descent trial. The left horizontal force increased in the direction of descent, while the right arm force was applied in the opposite direction; this pattern persisted until right foot contact at approximately $1.75 \mathrm{~s}$. The application of horizontal force on each rail in opposite directions from one another indicates that the participant used his arms to control trunk rotation. A similar compensation in the horizontal direction was not observed at the higher duty cycle. This force profile indicated that the participant was less comfortable with the speed of descent at the lower duty cycle, and thus he utilized his upper body to help guide his lower-limb motion during the latter half of CL. This conclusion was supported by the increased vertical ground reaction force on the top

Table.

Average angular range, duration, and angular velocity of trailing (left) limb during controlled lowering phase of descent at two variable impedance knee mechanism (VIKM) duty cycle levels. Number in parentheses is one standard deviation.

\begin{tabular}{|c|c|c|c|c|c|}
\hline $\begin{array}{l}\text { Damper Duty } \\
\text { Cycle (\%) }\end{array}$ & $\begin{array}{c}\text { Average VIKM } \\
\text { Torque Output } \\
\text { (Nm) }\end{array}$ & $\begin{array}{c}\text { Angle Range } \\
\left({ }^{\circ}\right)\end{array}$ & $\begin{array}{c}\text { Controlled Lowering } \\
\text { Duration (s) }\end{array}$ & $\begin{array}{l}\text { Mean Angular } \\
\text { Velocity ( } \% / \mathrm{s})\end{array}$ & $\begin{array}{l}\text { Peak Angular } \\
\text { Velocity }(\%)\end{array}$ \\
\hline$\overline{51.2}$ & 47 & $89.60(1.30)$ & $1.01(0.14)$ & $90.0(11.7)$ & $186.7(6.7)$ \\
\hline$p$-Value & - & 0.94 & 0.04 & 0.03 & 0.02 \\
\hline
\end{tabular}



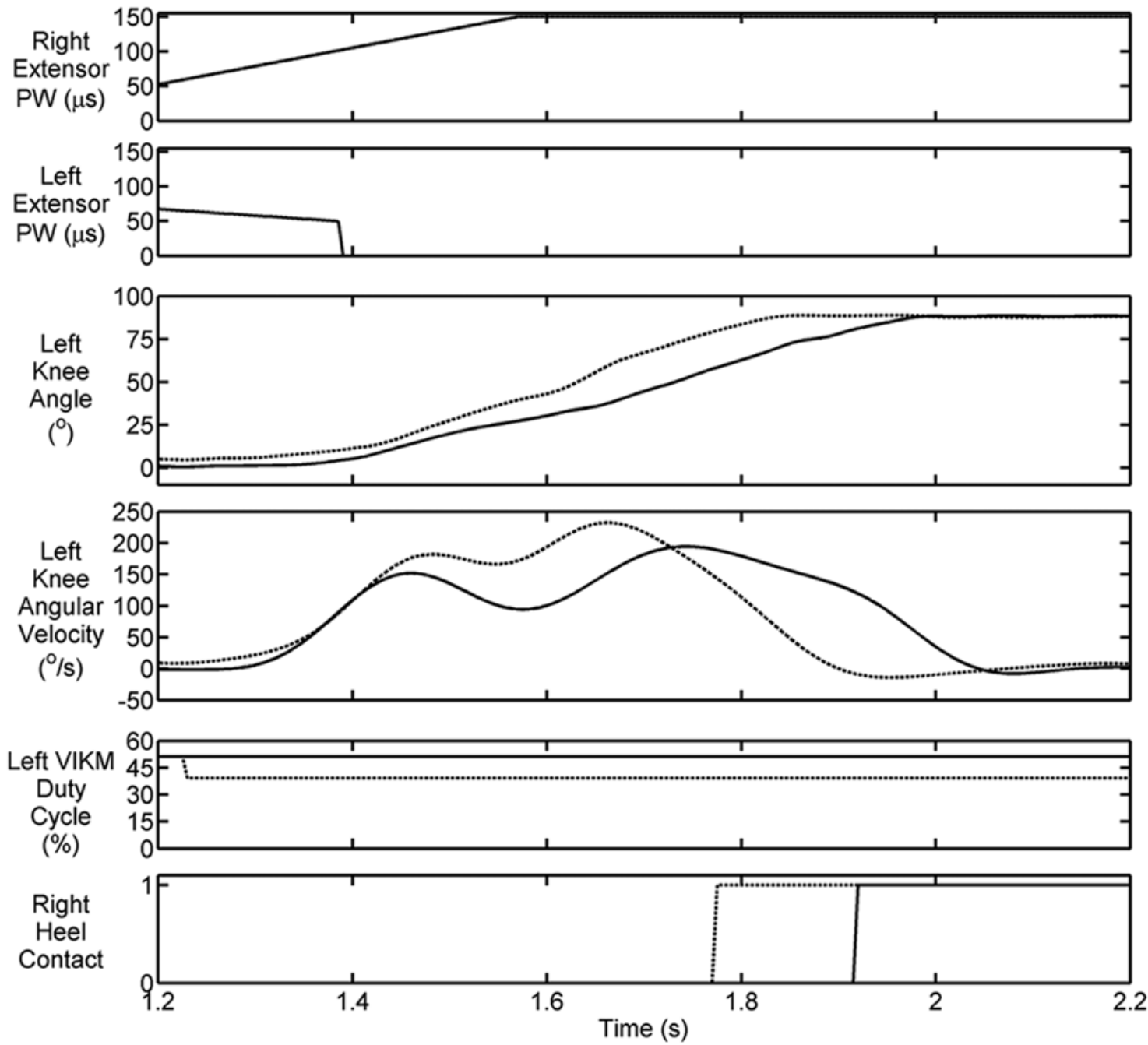

Figure 6.

Knee angle and angular velocity during typical controlled lowering phase of descent for two duty cycle levels, $51.2 \%$ (solid line) and $39.2 \%$ (dashed line). Trailing limb knee extensors are deactivated to allow variable impedance knee mechanism (VIKM) control of knee angle. $\mathrm{PW}=$ pulse width.

plate during CL at the higher duty cycle (Figure 8). Furthermore, the impulsive forces experienced immediately after foot contact were lower in both directions for the higher duty cycle. The total distribution of body weight support is approximately equal once a static posture is reached at $2.2 \mathrm{~s}$.

\section{DISCUSSION}

Previous studies report that control of stair descent via deactivation of hip and knee extensors requires 70 percent body weight support by the upper limbs [6] and that FNS users feel insecure during descent [8]. It has been postulated that insecurity during descent is a result of obstructed vision of the step below, but we hypothesize that lack of knee flexion control during the lowering phase is the primary contributor to discomfort. The results of this feasibility study demonstrate the ability of the VIKM orthosis to regulate knee flexion and control lowering speed through adjustment of VIKM duty cycle. By careful selection of duty cycle, knee flexion velocity during the initial phase of lowering remained below $150 \%$ s. This controlled speed was attained with almost no upper-limb force applied to the 

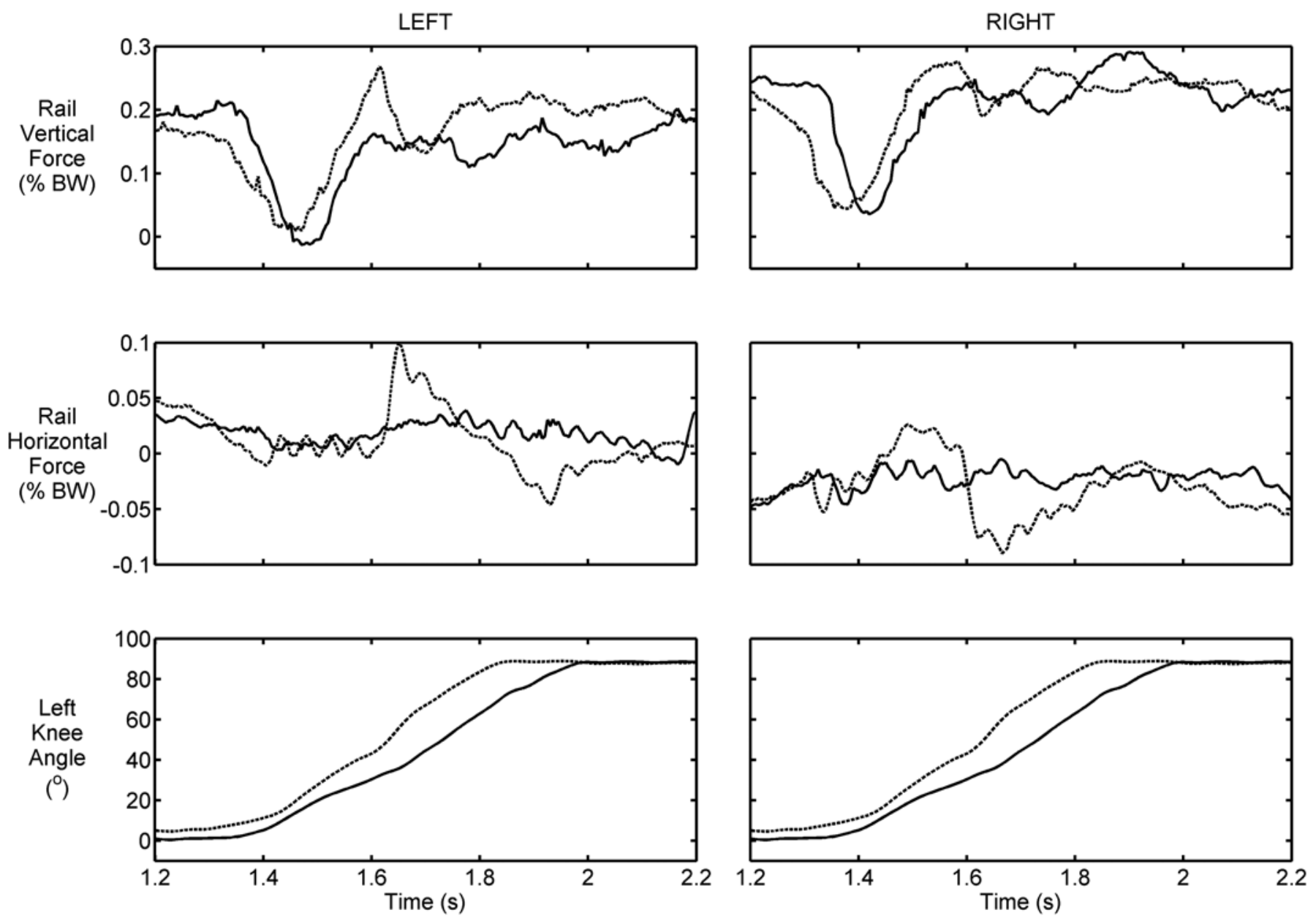

Figure 7.

Typical left and right hand rail vertical and horizontal forces (normalized by body weight [BW]) and knee angle during controlled lowering at high (solid line) and low (dashed line) variable impedance knee mechanism duty cycles.

handrails. As the leading limb neared the lower step, arm support resumed, but at a maximum level of approximately 40 to 45 percent body weight, a significant reduction compared with previous studies of FNS-only descent. The slower and smoother descent resulted in a vertical ground reaction force of 60 percent of body weight on the leading limb, a significant reduction from previously reported values [6]. The reduced load and slower descent were more acceptable to the user, who was able to differentiate between the two duty cycles following the descent without prior knowledge of them. Furthermore, the reduction in upper-limb support requirement allowed the user to tilt his trunk forward during descent, increasing vision of the step below, while the slower descent velocity increased the reliability of foot placement. These secondary effects likely contributed to the participant feeling safer during descent with the VIKM-HNP.

A key component of the stair descent maneuver is the muscle stimulation pattern (Figure 2). This pattern was conceived using previously established methods [2] and refined during calibration trials before the experiments took place. Prior to descent, the user's knees were extended by stimulation, and the ankles were locked by the AFO. But the user was able to support some body weight through his arms while flexing his trunk to view the step below. When the leading limb was lifted off the ground, this posture resulted in the ground reaction vector passing anterior to the stance knee, causing a slight extension moment. Thus, knee flexion for controlled lowering was initiated by unloading the arms and ramping off trailing 

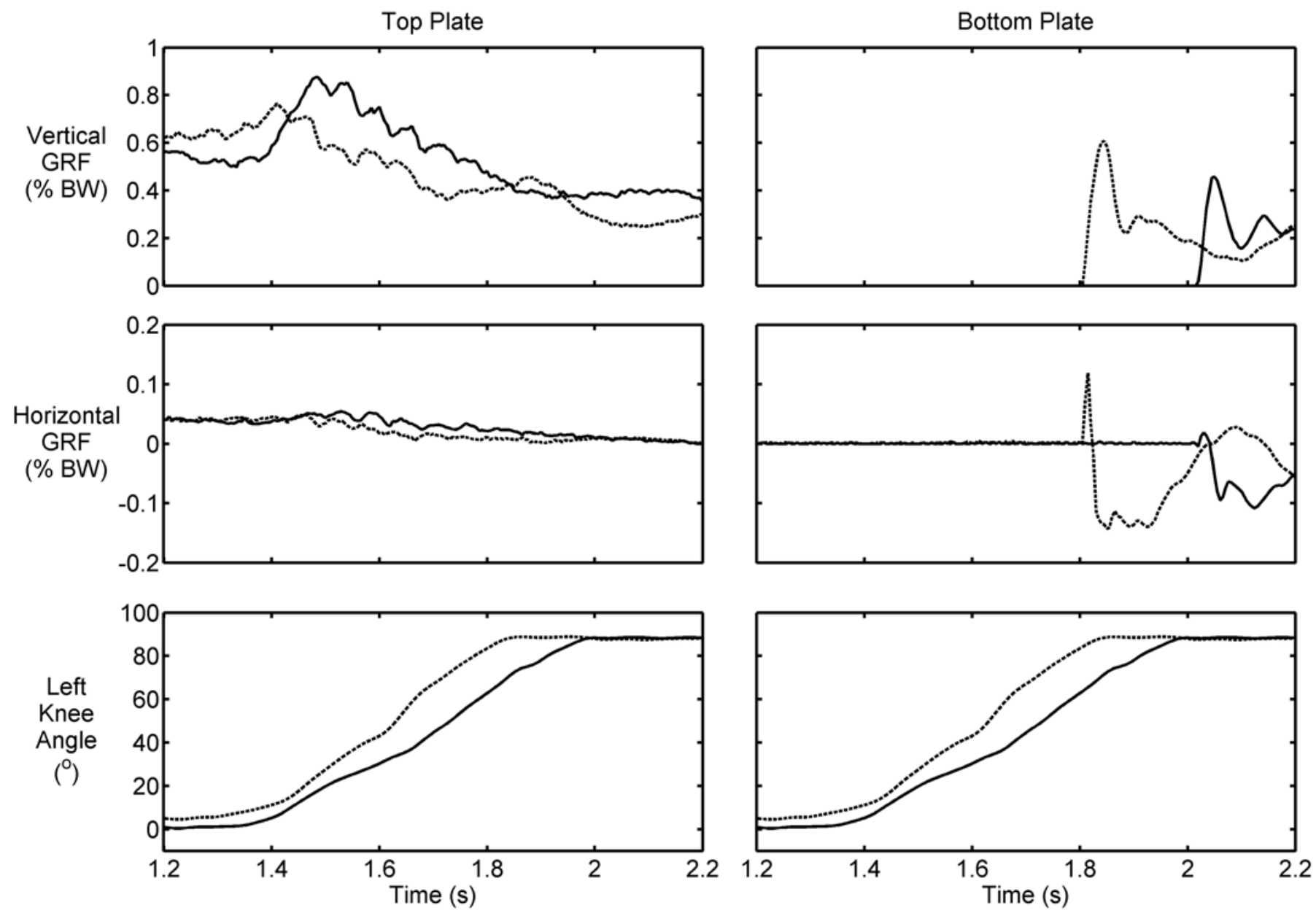

Figure 8.

Typical top and bottom plate ground reaction forces (GRFs) (normalized by body weight [BW]) and knee angle measured during controlled lowering at high (solid line) and low (dashed line) variable impedance knee mechanism duty cycle.

limb knee extensors. By design, the VIKM was only activated to control lowering after the leading limb knee extensors were ramped on (Figure 6), and thus, the relative timing between ramping down left knee extensors and ramping up right knee extensors was crucial.

The user-preferred VIKM duty cycle of 51.2 percent provided approximately $47 \mathrm{Nm}$ of resistive torque, imposed as an extensor moment of force at $45^{\circ}$ knee angle [16]. This equated to a normalized moment of approximately $0.63 \mathrm{Nm} / \mathrm{kg}$ provided during descent, a value that falls within the recently reported range of 0.40 to $1.1 \mathrm{Nm} / \mathrm{kg}$ of knee extensor torque required during the controlled lowering phase of normal stair descent [14,1819,24-25]. Note that the values in the literature were observed in step-over-step stair descent, whereas this study employed a step-by-step strategy. At first glance, our results appear in conflict with the postulation that step-by-step descent can decrease knee moment loading by up to 50 percent [11]. However, the confounding factor of a locked ankle joint in our experiments limited comparisons between normal values and those we observed. We observed flexion during the controlled lowering phase to knee angles at least two times as large as published studies of step-over-step descent. This increased knee flexion occurred because of the ankle restraint. Enhanced knee flexion significantly increased the knee moment arm and thus the level of resistive torque necessary to support the body during descent. The average power absorbed by the VIKM during controlled lowering was approximately $1.66 \mathrm{~W} / \mathrm{kg}$, which was 
larger than the reported range of 0.25 to $1.5 \mathrm{~W} / \mathrm{kg}$ $[14,18,26]$ despite the more conservative step-by-step strategy. In normal descent, the ankle provides significant power absorption (up to $2 \mathrm{~W} / \mathrm{kg}$ ) during lowering via plantar flexor muscles [26]. The VIKM-HNP does not activate plantar flexors but instead locks the ankle, a combination that inhibits power absorption capacity. This responsibility was passed on to the knee joint, where the active VIKM orthosis absorbed the additional power.

The VIKM is capable of supporting body weight in single stance, which would allow left (trailing limb) knee extensors to be turned off during state 1 . This would reduce the stimulation duty cycle and potentially delay fatigue. Similar savings could be achieved in state 3, during which right (leading limb) knee extensor stimulation could be turned off while the VIKM supports body weight. But the goal of the current study was to assess the ability of the VIKM to regulate lowering and facilitate step-by-step stair descent. Thus, knee extensor stimulation was used during single support phases to simplify the control scheme. Future implementations can include resting of knee extensors during these states, which could prolong use of the system by delaying muscle fatigue [6]. Moreover, an additional period for bilateral knee extensor rest could be added via introduction of a new double support state (with feet on two levels) between states 2 and 3, during which time both left and right VIKMs would be active to provide support at the knees.

While the VIKM orthosis showed an ability to regulate body lowering during trailing limb single support in place of stimulation, some limitations of FNS-driven swing motion during stair descent persisted, consistent with previously reported studies [5-8]. Namely, hip flexion during leg off and leg pull through were not strong enough to reliably achieve limb trajectory observed in nondisabled individuals during stair descent. Stair descent in nondisabled individuals requires hip flexion moments of approximately $0.10 \mathrm{Nm} / \mathrm{kg}$ and $0.52 \mathrm{Nm} / \mathrm{kg}$ during leg off and leg pull through, respectively [19]. The additional weight of the VIKM-HNP orthosis exacerbated these requirements. During LO, FNS must flex the hip and then maintain this flexion against gravity to allow knee extension for step clearance. LP necessitates hip and knee flexion to lift the trailing limb from the step. The torque that can be attained from stimulating the implanted hip flexor muscles (tensor fasciae latae and sartorius) with intramuscular electrodes is approximately 36 percent of normal near neutral posture, and the percentage decreases as hip flexion angle increases [27].
Thus, the torque generation capability of stimulated hip flexors in the VIKM-HNP muscle set was compromised during $\mathrm{LO}$ and $\mathrm{LP}$, when average hip angles were approximately $30^{\circ}$ and $50^{\circ}$, respectively. As a result, the participant was unable to consistently achieve the requisite hip flexion to lift the limb from the step during the first part of LP, resulting in the trunk rotation compensatory measures described previously. These hip flexion limitations and resultant compensatory measures inhibited fluid stair descent (Figure 5). In particular, delayed right (leading limb) hip flexion was observed during LO. Also, synergistic left (trailing limb) hip and knee flexion, followed by synergistic extension, was not observed during LP. Instead, hip extension mediated by trunk tilt and transverse rotation occurred first, followed by knee extension. These alterations of swing limb motion did not affect the ability of the VIKM to regulate lowering of the body from step to step or impede the participant from descending stairs with the VIKM-HNP. For safety, a spotter assured foot-step clearance during LO and LP.

The design of an effective neuroprosthetic intervention for stair descent must overcome this challenge. Improved implantation techniques of hip flexors [28] and advanced nerve cuff stimulation technology [29] offer potential increases in hip flexion moment compared with the intramuscular strategy employed in the VIKM-HNP system presented here. Specifically, recruitment of major hip flexors such as iliopsoas could improve hip flexion during LO and LP. Additional muscles can be added to the FNS system in the future to achieve higher flexion moments at the hip, but even then there will be a limitation on the hip moment generated by FNS in this flexed posture [27]. Another strategy is to incorporate a powered actuator into the orthosis to assist in hip flexion as needed when adequate torque is not available from stimulated muscle. Alternatively, an active ankle component could be incorporated into the HNP to provide plantar flexion assistance for push off. One solution would be to include an actuator at the ankle; however, the additional inertia from placing a powered mechanism on the distal portion of the limb would further impede hip and knee flexion during LO and LP (i.e., after the foot has left the ground). A better solution may be to implant electrodes for plantar and dorsiflexion stimulation, which could assist hip flexors during push-off and provide enhanced foot clearance during swing. Either way, future development of systems for stair descent should account for the increased flexion demands in their design. 
The experiments presented here demonstrate that the VIKM-HNP is able to effectively control stair descent in one individual with statistically significant differences in descent velocity between different VIKM settings. These findings demonstrate feasibility of achieving forward stair descent with an HNP driven by an FNS system designed for standing and walking. However, studies with additional participants, including varying levels of injury, are required to validate this approach across the full spectrum of individuals with thoracic-level SCI. Our future work will incorporate these larger scale studies.

\section{CONCLUSIONS}

FNS systems for stair descent suffer from two major drawbacks that limit their implementation: the inability of stimulation to achieve the requisite flexion torque for limb advancement and the inability of stimulated knee extensors to eccentrically contract during controlled lowering. The objective of this study was to evaluate the ability of a novel VIKM-HNP to substitute for the function of eccentric knee extensor and plantar flexor muscle contractions to absorb power and regulate body lowering to the next step without unduly loading the upper limbs. The results indicated that the VIKM-HNP and associated control system were able to achieve this performance and restore forward stair descent at controllable speeds that were comfortable for the user. Future work will focus on expansion of the current VIKM-HNP to aid in swing limb motion to restore reliable forward stair descent to users with paralysis from SCI.

\section{ACKNOWLEDGMENTS}

\author{
Author Contributions: \\ Study concept and design: T. C. Bulea, R. Kobetic, R. J. Triolo. \\ Acquisition of data: T. C. Bulea, R. Kobetic, M. L. Audu, \\ J. R. Schnellenberger. \\ Analysis and interpretation of data: T. C. Bulea, R. Kobetic, \\ M. L. Audu, R. J. Triolo. \\ Drafting of manuscript: T. C. Bulea, R. J. Triolo. \\ Critical revision of manuscript for important intellectual content: \\ T. C. Bulea, R. Kobetic, M. L. Audu, J. R. Schnellenberger, G. Pinault, \\ R. J. Triolo. \\ Statistical analysis: T. C. Bulea. \\ Obtained funding: R. Kobetic, R. J. Triolo. \\ Administrative, technical, or material support: R. Kobetic, \\ M. L. Audu, J. R. Schnellenberger, G. Pinault, R. J. Triolo.
}

Financial Disclosures: The authors have declared that no competing interests exist.

Funding/Support: This material was based on work supported in part by the Department of Veterans Affairs, Rehabilitation Research and Development Service (grants A6404-R and B0608-R) and the Department of Defense, Congressionally Directed Medical Research Program (grant PR043074). T. C. Bulea was also supported in part by the National Institutes of Health/National Institute of Arthritis and Musculoskeletal and Skin Diseases (grant T32 AR007505).

Additional Contributions: We thank Dr. Curtis S. To and Kevin Foglyano for their advice and contribution. Dr. Thomas C. Bulea is now with the Functional \& Applied Biomechanics Section at the National Institutes of Health.

Institutional Review: The volunteer signed a consent form approved by the institutional review board of the Louis Stokes Cleveland Department of Veterans Affairs Medical Center.

Participant Follow-up: The authors plan to inform the participant of the publication of this study.

\section{REFERENCES}

1. Bajd T, Kralj A, Turk R, Benko H, Sega J. The use of a four-channel electrical stimulator as an ambulatory aid for paraplegic patients. Phys Ther. 1983;63(7):1116-20. [PMID:6602994]

2. Kobetic R, Marsolais EB. Synthesis of paraplegic gait with multichannel functional neuromuscular stimulation. IEEE Trans Rehabil Eng. 1994;2(2):66-79.

http://dx.doi.org/10.1109/86.313148

3. Gallien P, Brissot R, Eyssette M, Tell L, Barat M, Wiart L, Petit H. Restoration of gait by functional electrical stimulation for spinal cord injured patients. Paraplegia. 1995; 33(11):660-64. [PMID:8584301] http://dx.doi.org/10.1038/sc.1995.138

4. Graupe D, Kohn KH. Functional neuromuscular stimulator for short-distance ambulation by certain thoracic-level spinal-cord-injured paraplegics. Surg Neurol. 1998;50(3): 202-7. [PMID:9736079] http://dx.doi.org/10.1016/S0090-3019(98)00074-3

5. Kobetic R, Carroll SG, Marsolais EB. Paraplegic stair climbing assisted by electrical stimulation. Proceedings of the 39th Annual Conference of Engineering in Medicine and Biology; 1986; p. 265.

6. Kobetic R, Marsolais EB, Samame P, Borges G. The next step: Artificial walking. In: Rose J, Gamble JG, editors. Human walking. 2nd ed. Baltimore (MD): Williams \& Wilkins; 1994. p. 225-52.

7. Rudel D, Bajd T, Kralj A, Benko H. Crutch, staircase railing and foot-floor forces during paraplegic's stair climbing. In: Bergmann G, Kölbel R, Rohlmann A, editors. Biomechanics: Basic and applied research. Boston (MA): Nijhoff; 1987. p. 551-56. 
8. Fuhr T, Quintern J, Schmidt G. Stair ascending and descending with the cooperative neuroprosthesis WALK! Neuromodulation. 2003;6(1):57-67. [PMID:22150914] http://dx.doi.org/10.1046/j.1525-1403.2003.03007.x

9. Cordo PJ, Rymer WZ. Motor-unit activation patterns in lengthening and isometric contractions of hindlimb extensor muscles in the decerebrate cat. J Neurophysiol. 1982;47(5):782-96. [PMID:7086469]

10. Triolo RJ, Robinson DE, Betz RR. Force-velocity and length-tension properties of stimulated human quadriceps muscle in spinal cord injured children. Proceedings of the Annual International Conference of the IEEE Engineering in Medicine and Biology Society; 1989 Nov 9-12; Seattle, WA. p. 967-68.

11. Andriacchi TP, Andersson GB, Fermier RW, Stern D, Galante JO. A study of lower-limb mechanics during stairclimbing. J Bone Joint Surg Am. 1980;62(5):749-57. [PMID:7391098]

12. McFadyen BJ, Winter DA. An integrated biomechanical analysis of normal stair ascent and descent. J Biomech. 1988;21(9):733-44. [PMID:3182877] http://dx.doi.org/10.1016/0021-9290(88)90282-5

13. Costigan PA, Deluzio KJ, Wyss UP. Knee and hip kinetics during normal stair climbing. Gait Posture. 2002;16(1):31-37. [PMID:12127184] http://dx.doi.org/10.1016/S0966-6362(01)00201-6

14. D Beaulieu FG, Pelland L, Robertson DG. Kinetic analysis of forwards and backwards stair descent. Gait Posture. 2008;27(4):564-71. [PMID:17825566] http://dx.doi.org/10.1016/j.gaitpost.2007.07.010

15. Bulea TC, Kobetic R, To CS, Audu ML, Schnellenberger JR, Triolo RJ. A variable impedance knee mechanism for controlled stance flexion during pathological gait. IEEE/ ASME Trans Mechatron. 2012;17(5):822-32. http://dx.doi.org/10.1109/TMECH.2011.2131148

16. Bulea TC, Kobetic R, Audu ML, Schnellenberger JR, Triolo RJ. Finite state control of a variable impedance hybrid neuroprosthesis for locomotion after paralysis. IEEE Trans Neural Syst Rehabil Eng. 2013;21(1):141-51.

[PMID:23193320] http://dx.doi.org/10.1109/TNSRE.2012.2227124

17. Bulea TC, Kobetic R, Audu ML, Triolo RJ. Stance controlled knee flexion improves stimulation driven walking after spinal cord injury. J Neuroeng Rehabil. 2013;10:68. [PMID:23826711] http://dx.doi.org/10.1186/1743-0003-10-68

18. Riener R, Rabuffetti M, Frigo C. Stair ascent and descent at different inclinations. Gait Posture. 2002;15(1):32-44. [PMID:11809579] http://dx.doi.org/10.1016/S0966-6362(01)00162-X

19. Protopapadaki A, Drechsler WI, Cramp MC, Coutts FJ, Scott OM. Hip, knee, ankle kinematics and kinetics during stair ascent and descent in healthy young individuals. Clin Biomech (Bristol, Avon). 2007;22(2):203-10.

[PMID:17126461]

http://dx.doi.org/10.1016/j.clinbiomech.2006.09.010

20. Mortimer JT, Kaufman D, Roessman U. Intramuscular electrical stimulation: Tissue damage. Ann Biomed Eng. 1980;8(3):235-44. [PMID:7224246]

http://dx.doi.org/10.1007/BF02364479

21. Carroll SG, Triolo RJ, Chizeck HJ, Kobetic R, Marsolais EB. Tetanic responses of electrically stimulated paralyzed muscle at varying interpulse intervals. IEEE Trans Biomed Eng. 1989;36(7):644-53. [PMID:2787276] http://dx.doi.org/10.1109/10.32096

22. Kobetic R, To CS, Schnellenberger JR, Audu ML, Bulea TC, Gaudio R, Pinault G, Tashman S, Triolo RJ. Development of hybrid orthosis for standing, walking, and stair climbing after spinal cord injury. J Rehabil Res Dev. 2009; 46(3):447-62. [PMID:19675995] http://dx.doi.org/10.1682/JRRD.2008.07.0087

23. Audu ML, To CS, Kobetic R, Triolo RJ. Gait evaluation of a novel hip constraint orthosis with implication for walking in paraplegia. IEEE Trans Neural Syst Rehabil Eng. 2010; 18(6):610-18. [PMID:20378478]

http://dx.doi.org/10.1109/TNSRE.2010.2047594

24. Reeves ND, Spanjaard M, Mohagheghi AA, Baltzopoulos $\mathrm{V}$, Maganaris CN. The demands of stair descent relative to maximum capacities in elderly and young adults. J Electromyogr Kinesiol. 2008;18(2):218-27. [PMID:17822923] http://dx.doi.org/10.1016/j.jelekin.2007.06.003

25. Novak AC, Brouwer B. Sagittal and frontal lower limb joint moments during stair ascent and descent in young and older adults. Gait Posture. 2011;33(1):54-60.

[PMID:21036615]

http://dx.doi.org/10.1016/j.gaitpost.2010.09.024

26. Duncan JA, Kowalk DL, Vaughan CL. Six degree of freedom joint power in stair climbing. Gait Posture. 1997;5(3):204-10. http://dx.doi.org/10.1016/S0966-6362(96)01086-7

27. Kobetic R, Marsolais EB, Miller PC. Function and strength of electrically stimulated hip flexor muscles in paraplegia. IEEE Trans Rehabil Eng. 1994;2(1):11-17. http://dx.doi.org/10.1109/86.296347

28. Nandurkar S, Marsolais EB, Kobetic R. Percutaneous implantation of iliopsoas for functional neuromuscular stimulation. Clin Orthop Relat Res. 2001;389(389):210-17. [PMID:11501813] http://dx.doi.org/10.1097/00003086-200108000-00030

29. Schiefer MA, Triolo RJ, Tyler DJ. A model of selective activation of the femoral nerve with a flat interface nerve electrode for a lower extremity neuroprosthesis. IEEE Trans Neural Syst Rehabil Eng. 2008;16(2):195-204. [PMID:18403289] http://dx.doi.org/10.1109/TNSRE.2008.918425 
Submitted for publication December 2, 2013. Accepted in revised form April 30, 2014.

This article and any supplementary material should be cited as follows:

Bulea TC, Kobetic R, Audu ML, Schnellenberger JR, Pinault G, Triolo RJ. Forward stair descent with hybrid neuroprosthesis after paralysis: Single case study demonstrat- ing feasibility. J Rehabil Res Dev. 2014;51(7):1077-94.

http://dx.doi.org/10.1682/JRRD.2013.12.0257

ResearcherID/ORCID: Musa L. Audu, PhD: H-32512014; Ronald J. Triolo, PhD: H-3712-2014

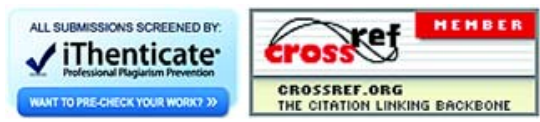


